\title{
INFLUENCIA DE LA REVEGETACIÓN CON ESPECIES NATIVAS Y LA INCORPORACIÓN DE MATERIA ORGÁNICA EN LA RECUPERACIÓN DE PASTIZALES DEGRADADOS
}

\section{INFLUENCE OF REVEGETATION USING NATIVE SPECIES AND THE INCORPORATION OF ORGANIC MATTER IN THE RECOVERY OF DEGRADED RANGELANDS}

\author{
Raúl E. Tácuna ${ }^{1}$, Lucrecia Aguirre ${ }^{2}$ y Enrique R. Flores ${ }^{2}$
}

\begin{abstract}
Resumen
Se realizó una investigación con enfoque participativo (IEP) en un ecosistema montañoso de la Cordillera Blanca de Perú dominado por pajonales de condición pobre. El objetivo fue determinar el efecto de la revegetación con esquejes de dos gramíneas nativas claves (Festuca humilior y Calamagrostis macrophylla) y la adición de materia orgánica en forma de orina y estiércol de ovino sobre la recuperación del estatus ecológico de un pastizal de condición pobre. El diseño experimental fue completamente al azar con arreglo factorial 2 especies x 2 niveles de adición, con y sin materia orgánica. El estudio duró un año, periodo durante el cual se estudiaron los cambios en la vegetación (vigor y mortalidad), la función hidrológica (tasa de infiltración y contenido de humedad del suelo) y el estatus ecológico. Los resultados revelaron que los esquejes de Festuca humilior superaron a los de Calamagrostis macrophylla en vigor (20 595.3 vs 13 295.9 , cc) y exhibieron menores tasas de mortalidad (36.0 vs $51.5 \%$ ) y estas respuestas fueron potenciadas con la adición de materia orgánica. Los mismos patrones se observaron cuando se evaluó la respuesta hidrológica, a través de la tasa de infiltración $(0.14 \mathrm{vs} 0.11 \mathrm{~cm} / \mathrm{min}$.) y contenido de humedad (21.8 vs 19.0\%). Los resultados indican también que la mejora en el estado de la vegetación y la función hidrológica habrían contribuido a la mejora observada en el estatus ecológico de las parcelas tratadas (condición regular) con respecto a las tierras de usufructo comunal (condición pobre). Se concluye que la revegetación de pastizales pobres con esquejes de gramíneas nativas y la incorporación de materia orgánica en forma de orina y estiércol de ovino es una estrategia efectiva para mejorar la condición y función hidrológica del pastizal. Se recomienda evaluar el impacto de la revegetación a nivel del productor en combinación con sistemas de pastoreo de descanso y pastoreo diferido para asegurar la sostenibilidad de programas de rehabilitación de pastizales degradados.
\end{abstract}

Palabras clave: Degradación, revegetación, esquejes, materia orgánica, condición, vigor, infiltración.

\footnotetext{
Abstract

Participatory-Oriented Research was conducted in a mountain ecosystem, dominated by grassland in poor condition, in the Cordillera Blanca of Peru. The objective of the experiment was to determine the effect of revegetation with plantlets of two key native grasses (Festuca humilior and Calamagrostis macrophylla) and the addition of organic matter in the form of sheep urine and manure on the recovery of the ecological status of poor condition rangeland. The experimental design was completely randomized with a factorial arrangement of 2 species $\mathrm{x} 2$ with and without the addition of organic matter. The study lasted a year, period during which the changes in vegetation (vigor and mortality), the hydrologic function (rate of infiltration and soil moisture content) and ecological status were studied. The results of the study revealed that the vigor of the Festuca humilior plantlets was greater than that of its Calamagrostis macrophylla counterpart (20 $595.3 \mathrm{cc}$ vs. $13295.9 \mathrm{cc})$ and that they exhibited lower mortality rates $(36.0 \%$ vs. $51.5 \%)$. The results also showed that these responses were enhanced when organic matter was added. The same patterns were observed when the hydrologic response, considering the rate of infiltration $(0.14 \mathrm{~cm} / \mathrm{min}$ vs. $0.11 \mathrm{~cm} / \mathrm{min})$ and moisture content $(21.8 \%$ vs. $19.0 \%)$, was evaluated. The results also indicate that the improvement in vegetation condition and hydrologic function must have contributed to the improvement in the rangeland ecological status of the treated plots (regular condition) in relation to the communal land (poor condition). Therefore, one can conclude that the
} 
revegetation of poor rangeland with plantlets of native species is an effective strategy to improve its condition and hydrologic function. Assessing the impact of revegetation at the producer level in combination with rest-rotation and a deferred-rotation grazing system is recommended to ensure the sustainability of degraded range rehabilitation programs.

Key words: Degradation, rangeland, revegetation, plantlets, organic matter, condition, vigor, infiltration.

\section{Introducción.}

Una importante proporción de las tierras de pastoreo de manejo comunal en los Andes peruanos se encuentran en condición pobre (62\%) debido principalmente al sobrepastoreo y la aplicación de malas prácticas de manejo (Flores, 1999). La condición del pastizal es un término ecológico que hace referencia al estado de salud de los pastizales con referencia al potencial que estos podrían alcanzar con respecto a su estado de comunidad madura. La sostenibilidad de los ecosistemas de pastizal depende en gran medida del equilibrio existente entre el estatus del agua, el suelo y la vegetación. En pastizales de condición pobre este equilibrio puede ser alterado por cuanto la cubierta vegetal es escasa y en consecuencia el suelo se encuentra pobremente protegido, lo cual afecta la fertilidad del suelo y por ende la productividad del pastizal (Pyke et al., 2002).

La degradación de los pastizales es evidenciada por el cambio detrimental de las características de la vegetación y la función hídrica, principalmente en la reducción de la cobertura vegetal, la desaparición de las especies botánicas claves y la disminución de la materia orgánica, la tasa de infiltración y el estatus de humedad del suelo (Whitford, 1995). El deterioro de la condición de los pastizales tiene un impacto negativo en el valor, servicios $y$ beneficios ambientales que brindan estos ecosistemas (Petersen \& Stringham, 2008). Frente a este panorama existe preocupación por llevar a la práctica estrategias de mejora de pastizales que resulten viables para la rehabilitación de ecosistemas degradados desde el punto de vista económico y ecológico, con la finalidad de agregar sostenibilidad a los sistemas de producción pecuarios, pues cuando la condición y productividad del pastizal se mejora, se incrementa la estabilidad y resistencia de los ecosistemas a las perturbaciones (Krogh et al., 2002).

Rehabilitar un pastizal significa elevar su condición, productividad y capacidad de carga, para lo cual los manejadores de pastizales hacen uso de las estrategias de mejora de la condición que pueden ser clasificadas en: extensivas e intensivas, en función a criterios como el nivel de riesgo, costo de inversión, nivel de producción, rentabilidad y nivel de tecnología (Herbel, 1983). La adición de materia orgánica, en la forma de estiércol y orina de ovino, y la revegetación con semillas o material vegetativo de especies nativas claves son prácticas de mejora para recuperar pastizales degradados (Flores, 1999; Zhang et al.,
2011; Wang et al., 2012). Sin embargo, en Perú no se cuenta con información científica acerca de su efectividad en la restauración de los ecosistemas de pastizal degradados, bajo las condiciones ambientales de la Puna (Call \& Roundy, 1991; Krogh et al., 2002).

El objetivo del presente estudio fue evaluar el efecto de la revegetación con esquejes de dos especies de gramíneas nativas claves (Festuca humilior y Calamagrostis macrophylla) con y sin adición de materia orgánica sobre la dinámica de la revegetación, la función hídrica del suelo y el estatus ecológico de un pastizal de condición pobre. Las preguntas de investigación fueron ¿Cuál es el grado de la interacción entre la materia orgánica y la revegetación con esquejes?, ¿Son las especies elegidas adecuadas para la revegetación?, ¿En qué medida mejora la respuesta hidrológica del pastizal con la revegetación?

\section{Materiales y métodos.}

Área de Estudio

La presente investigación se realizó en Sillacancha, un área de usufructo comunal de la comunidad campesina Cordillera Blanca, en el distrito de Recuay, provincia de Recuay, en la región Ancash, a una altitud de $4100 \mathrm{msnm}$. La zona de estudio, de acuerdo con la descripción los Ecosistemas de los Andes del Norte y Centro pertenece a la clasificación Pajonal Altoandino Húmedo (Flores \& Naupari, 2011), caracterizada por presentar una temperatura promedio de $12^{\circ} \mathrm{C}$, con vientos fríos y secos y una precipitación anual promedio de $700 \mathrm{~mm}$ (Mamani, 2002). El tipo de pastizal es un pajonal con una cobertura vegetal del $51 \%$ y un $2 \%$ de mantillo, dominado por una asociación de Calamagrostis macrophylla - Stipa brachyphylla - Scirpus rigidus.

La condición del pastizal es pobre para ovinos y exhibe una franca tendencia al deterioro por el sobrepastoreo y mal manejo. Los suelos son superficiales y pedregosos, de una textura francoarenosa y presentan signos de erosión moderados. Estos suelos exhiben un $\mathrm{pH}$ de 4.53, una conductividad eléctrica de $0.11 \mathrm{dS} / \mathrm{m}$, la capacidad de intercambio catiónico de $9.12 \mathrm{meq} / 100 \mathrm{~g}$, con un contenido de fósforo de $3.4 \mathrm{ppm}$, potasio de $24 \mathrm{ppm}$ y un contenido de materia orgánica del $4.2 \%$ (Laboratorio de Análisis de Suelos y Aguas UNALM, 2011). El estudio tuvo la duración de un año, comprendido entre noviembre 2010 a noviembre 2011. 
El lugar tiene una larga historia de pastoreo pesado y se ubica en una posición topográfica plana o casi plana. El manejo de los campos de usufructo comunal por parte de la comunidad Cordillera Blanca se desarrolla a través de "manadas", la cual es la base social de manejo de la ganadería en la comunidad y se trata de grupos de familias que pastorean de forma cooperativa un determinado sector asignado por la comunidad, rotando entre sí las labores de pastoreo (1 a 2 meses por familia). No existe una división o cercado en los terrenos de usufructo comunal donde pastorean estacionalmente los animales y en función a la carga animal y la extensión del área ocupada por los ovinos, se ha estimado para el área de usufructo comunal un aporte de estiércol de $131.4 \mathrm{~kg} /$ ha /año (Rondán \& Chávez, 2014).

\section{Tratamientos Experimentales}

Se aplicaron cuatro tratamientos experimentales que resultaron de la combinación de dos prácticas de mejora de pastizales, revegetación con esquejes de gramíneas nativas claves promisorias y adición de materia orgánica en la forma de orina y estiércol de ovino. Los tratamientos experimentales fueron: revegetación con Festuca humilior con adición de materia orgánica $(\mathrm{T} 1:$ Fehu $+\mathrm{MO})$, revegetación con Festuca humilior sin adición de materia orgánica (T2: Fehu), revegetación con Calamagrostis macrophylla con adición de materia orgánica (T3: Cama + MO) y revegetación con Calamagrostis macrophylla sin adición de materia orgánica (T4: Cama). Estos tratamientos fueron implementados cada uno en una parcela de evaluación de $25 \times 25 \mathrm{~m}$, debidamente cercadas y excluidas del usufructo comunal. Este estudio se realizó bajo condiciones de secano, es decir la única forma de suplementación de agua provino de la precipitación.

La incorporación de estiércol y orina de ovino se realizó a través de un majadeo (Rodríguez, 2006), para lo cual se procedió a ubicar un lote de 150 unidades ovino (UO) durante dos noches consecutivas en las parcelas que recibieron los tratamientos de adición de materia orgánica (T1 y $\mathrm{T} 3$ ). Este tiempo de permanencia en las parcelas fue suficiente para obtener un aporte estimado de $250 \mathrm{~kg}$ de estiércol de ovino (4 t/ha). Los esquejes de las especies nativas utilizadas para la revegetación sobre el tapiz natural fueron colectadas de áreas adyacentes y plantadas en hoyos a un metro de distancia entre sí, equivalente a una densidad de 1 esqueje $/ \mathrm{m}^{2}$. En adición, se realizaron evaluaciones en el área de usufructo comunal colindante a las parcelas de evaluación, con la finalidad de comparar el estatus ecológico de estas parcelas con la de los tratamientos.

\section{Parámetros Evaluados}

La respuesta del pastizal degradado a la aplicación de los tratamientos se evaluó a lo largo de un año a través de cinco parámetros, dos para determinar el efecto de la adición de la materia orgánica sobre el establecimiento de los esquejes y la dinámica de la vegetación (porcentaje de mortalidad y vigor de esquejes), otros dos para estimar la respuesta hidrológica a la revegetación con esquejes (tasa de infiltración y contenido de humedad del suelo) y finalmente la condición del pastizal como indicador de la respuesta ecológica de cada tratamiento (Flores, 1996). Cabe resaltar que los monitoreos fueron realizados utilizando una metodología de la Investigación con Enfoque Participativo (IEP).

\section{a. Porcentaje de mortalidad y vigor de esquejes.}

Ambos parámetros fueron monitoreados cada dos meses y para ello previamente fueron seleccionados y marcados 30 esquejes de cada especie por tratamiento. La mortalidad de los esquejes fue estimada en porcentaje, mediante la apreciación visual y conteo directo del grado de prendimiento de cada esqueje marcado sobre el tapiz natural, donde la observación de esquejes senescentes y/o marchitos son considerados como "muertos" (Gillen \& Tate, 1993).

El vigor de los esquejes estuvo representado por la variación del volumen que estos adquirieron en cada momento de evaluación (Weaver \& Darlan, 1947). La obtención del volumen del esqueje fue calculado en función a las dimensiones del diámetro basal (diámetro de la corona), diámetro de la canopia (diámetro aéreo) y altura del esqueje a la hoja bandera, simulando las dimensiones de un tronco de cono invertido, bajo la siguiente relación:

Volumen del esqueje $=\left(\frac{D+d}{2}\right)^{2} \pi \mathrm{h}$

Dónde: D: diámetro basal

d: diámetro aéreo

h: altura

b.Tasa de infiltración y humedad del suelo.

La tasa de infiltración de agua en el suelo fue estimada mediante el empleo de un infiltrómetro realizando dos muestreos por tratamiento cada dos meses. Las mediciones fueron realizadas tomando lecturas cada 1,5 y 10 minutos para finalmente obtener el valor en el que la diferencial $\mathrm{cm} / \mathrm{min}$ es constante. Esto permitió desarrollar curvas de infiltración y estimar el valor promedio de infiltración por tratamiento (Bouwer, 1986; Hurtado, 2007).

La humedad del suelo se estimó cada dos meses mediante la extracción de 5 muestras de suelo por tratamiento a $15 \mathrm{~cm}$ de profundidad (Blake, 1965). Posteriormente, las muestras de suelo colectadas fueron secadas en una estufa a $105 \mathrm{C}^{\circ}$ por 24 horas y finalmente por diferencia de peso se calculó el contenido de humedad del suelo bajo la siguiente relación (Pierson et al., 2002):

$\%$ Humedad $=\frac{\text { Peso húmedo }- \text { Peso seco }}{\text { Peso húmedo }} \times 100$ 
c. Condición Ecológica del pastizal.

La condición ecológica del pastizal se estimó dos veces, antes de la aplicación de los tratamientos y al finalizar el estudio, en el área representativa de cada parcela experimental y usufructo comunal por el método de Transección lineal permanente (Parker, 1951). Las especies botánicas encontradas fueron clasificadas taxonómicamente y de acuerdo a su grado de deseabilidad para ovinos en deseables, poco deseables e indeseables (Flores, 1999). La composición florística se determinó expresando al $100 \%$ sólo a las especies vegetales encontradas con cada censo; es decir, no se incluyó a los demás elementos sin valor forrajero (Ratliff, 1993). Este parámetro, posteriormente permitió estimar la condición del pastizal con respecto a las tierras de usufructo comunal a partir de la siguiente ecuación (Flores, 1999):

Puntaje $(0-100 \%)=0.5(\% \mathrm{D})+0.2(\% \mathrm{IF})+0.2(\% \mathrm{CV})+0.1(\% \mathrm{IV})$

Donde:

D (\%): Porcentaje de especies deseables para ovinos.

IF (\%): Porcentaje de especies forrajeras.

CV(\%): Porcentaje de cobertura vegetal.

IV(\%): Porcentaje de índice de vigor de las plantas claves (Festuca humilior y Calamagrostis macrophylla).

\section{Diseño Experimental}

El diseño experimental utilizado fue un completamente al azar con arreglo factorial 2 x 2 , con cuatro tratamientos resultantes de la combinación de dos especies vegetales y dos niveles de adición de materia orgánica. Cada tratamiento fue implementado en parcelas de evaluación de $25 \times 25 \mathrm{~m}$ en las cuales se eligieron al azar 30 esquejes plantados a los cuales se les tomaron 6 sets de medidas repetidas por parcela, 2 por época lluviosa, transicional y seca. Estas medidas al ser tomadas dentro de una parcela constituyen pseudoréplicas (Hulbert, 1984). Para separar los promedios de los parámetros estudiados se utilizó una prueba diferencia límite de significancia, $\operatorname{DLS}(\alpha=0.05)$.

\section{Resultados y discusión.}

Los resultados revelan que la adición de materia orgánica, mejoró la respuesta adaptativa del esqueje al ecosistema de pastizal pobre, probablemente como resultado de la disponibilidad inmediata de los nutrientes suplementados por la orina $(\mathrm{N}, \mathrm{K})$ y mejora en las propiedades físicas del suelo, por lo que los resultados de la revegetación del pastizal fueron positivas (Snyman \& Du Preez, 2005). Por otro lado, el estiércol al permanecer sobre la superficie cumple un rol aislador (Jing et al., 2014; Moreno, 2015) para reducir los cambios bruscos de temperatura a nivel del suelo durante el día y la noche que son marcados en la zona altoandina, y esta reducción en la magnitud de los cambios de temperatura a nivel del suelo podría haber contribuido a un mejor establecimiento de los esquejes (Pierson et al., 2002; Petersen \& Stringham, 2008).

La elección de gramíneas promisorias para la revegetación, Festuca humilior y Calamagrostis macrophylla, fue acertada para llevar a cabo este experimento, por cuanto las gramíneas son las que presentan un mejor desempeño para la revegetación de pastizales degradados, por sus características adaptativas, mayor resistencia a las perturbaciones, facilidad de propagación y establecimiento en un tiempo relativamente corto (Smith et al., 1997). Al respecto, Montemayor et al. (2008) e Ilunga wa Ilunga et al. (2015) recomiendan que dentro de las características de las plantas para que pueden ser aprovechadas en programas de revegetación de pastizales degradados se incluyan: especies claves, dominantes, ciclo de vida perenne, alto crecimiento durante la época de lluvias, tolerante a las condiciones del clima, salinidad, acidez y comprobada supervivencia al transplante, aptitudes que pudimos comprobar con las dos especies promisorias seleccionadas para la ejecución del experimento. Sin embargo, es necesario señalar que el establecimiento de esquejes puede variar según el tipo de suelo y hábitos de enraizamiento (Schwinning \& Hooten, 2009).

Dinámica de la Revegetación

La adición de materia orgánica tuvo una influencia significativa $(\mathrm{P}<0.05)$ sobre la mortalidad de los esquejes trasplantados (Figura 1), la cual mantuvo una tendencia creciente al inicio de la evaluación para después estabilizarse. En ambas especies, se encontró que la magnitud de la mortalidad fue superior cuando los esquejes no recibieron la aplicación de la materia orgánica $(\mathrm{P}<0.01)$. Analizando el comportamiento entre especies, se encontró que la mayor mortalidad de esquejes ocurrió con Calamagrostis macrophylla en ambas situaciones, con y sin materia orgánica $(38.0$ y $65.0 \%$ respectivamente) en comparación con la Festuca humilior (31.0 y $41.0 \%$ respectivamente). Estos resultados son acordes con los trabajos realizados por Ghose (2001) y Burke (2008), quienes encontraron una mayor sobrevivencia al trasplante en los sitios de pastizal que recibieron materia orgánica como enmienda en comparación con campos que no recibieron ninguna adición de materia orgánica. Los autores proponen que la mayor sobrevivencia de esquejes se debe a que el agregado de materia orgánica al suelo aumentó el contenido de nutrientes del suelo, la capacidad de retención de agua del suelo y la biota del suelo, incluyendo mayor presencia de micorrizas. 

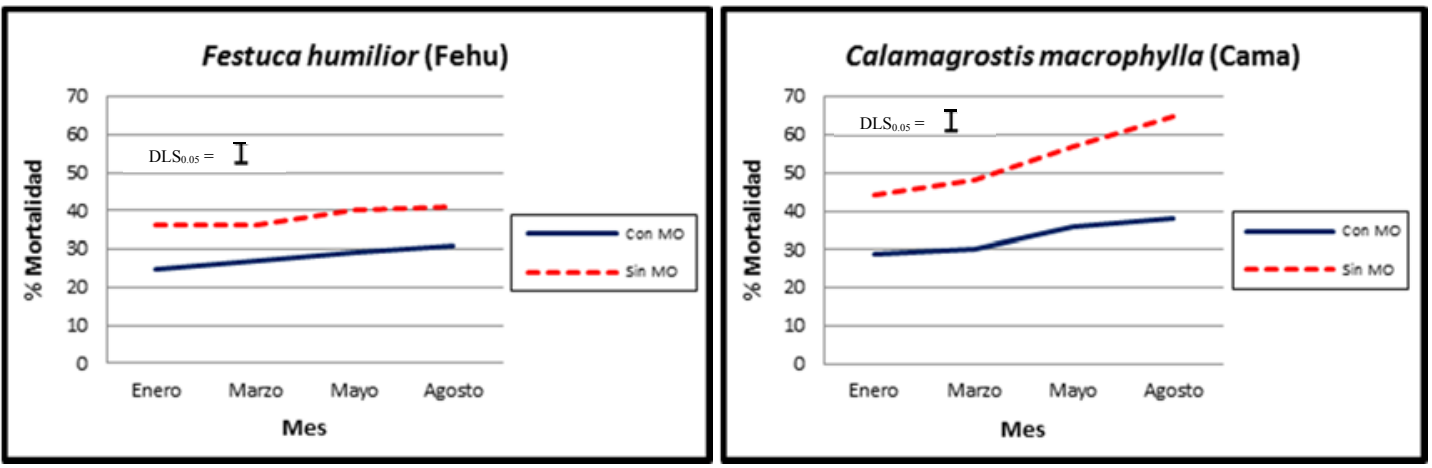

Figura 1. Mortalidad de esquejes con y sin materia orgánica.

La respuesta favorable al trasplante pudo estar asociada con la época de siembra, al inicio de la época lluviosa, tal como lo sugiere Commander et al. (2013), quienes concluyeron que la mejor época para la reintroducción de esquejes de plantas nativas es en el momento de alta precipitación, si se trata de un sistema secano. De este modo, la revegetación es viable, aprovechando para ello las condiciones naturales de humedad que favorecen el establecimiento de los esquejes (Allen, 1995; Burke, 2001). Asimismo, Abella et al. (2015) encontraron que al proporcionar suelo superficial de sistemas naturales rico en materia orgánica, sin la aplicación de riego adicional, se duplicó la sobrevivencia del trasplante, lo cual fue casi equivalente a las plantas que recibieron riego pero no la enmienda.

La sobrevivencia de los esquejes en el presente estudio puede haber sido favorecida por la técnica de revegetación empleada, la cual permitió el establecimiento de los esquejes sobre el tapiz natural. Hoag (2003), Steed \& DeWald (2003) y Montemayor et al., (2008) observaron que en estudios de trasplantes de plantas nativas a partir de esquejes, la utilización de su sustrato natural o tierra intacta, de tal manera que cubra la mayor densidad radicular, aumentó el éxito de establecimiento de las plantas. Este patrón pudo deberse, en parte, a la presencia de microorganismos beneficiosos a nivel de la raíz. De igual modo, Thomsen et al. (2005) encontraron que después de 9 meses de crecimiento, la biomasa de plantas trasplantadas con su sustrato natural fue mayor que las de plantas obtenidas de vivero.

Asimismo, dentro de la comunidad vegetal existen grupos de plantas que crecen juntas en coexistencia e interacciones específicas, situación que pudo favorecer aún más el éxito de la revegetación (Lawesson \& Oksanen, 2002). Tal como lo reportan Mola et al. (2011) y de la Riva et al. (2001) quienes concluyeron que la adición de materia orgánica mejoró las condiciones ambientales para el establecimiento de plántulas a través de la generación de micrositios adecuados, lo cual puede resultar en un proceso de facilitación para la introducción o regeneración posterior de nuevas especies dentro del micrositio establecido una vez que este mejoró (Méndez et al., 2008).

La adición de materia orgánica aceleró el crecimiento $(\mathrm{P}<0.05)$ y mejoró el volumen de los esquejes en ambas especies vegetales $(\mathrm{P}<0.05)$ (Figuras 2 y 3). En el caso de la Festuca humilior se encontraron alturas finales de 64.9 y $44.2 \mathrm{~cm}$, y volúmenes finales de esquejes de 26045.7 y 15144.9 cc para los tratamientos con y sin materia orgánica respectivamente. La misma tendencia se encontró en el caso de la Calamagrostis macrophylla, debido a que se encontraron alturas finales de 50.3 y $33.6 \mathrm{~cm}$, y volúmenes finales de esquejes de $\quad 18334.2$ y 8 757.7 cc para los tratamientos con y sin materia
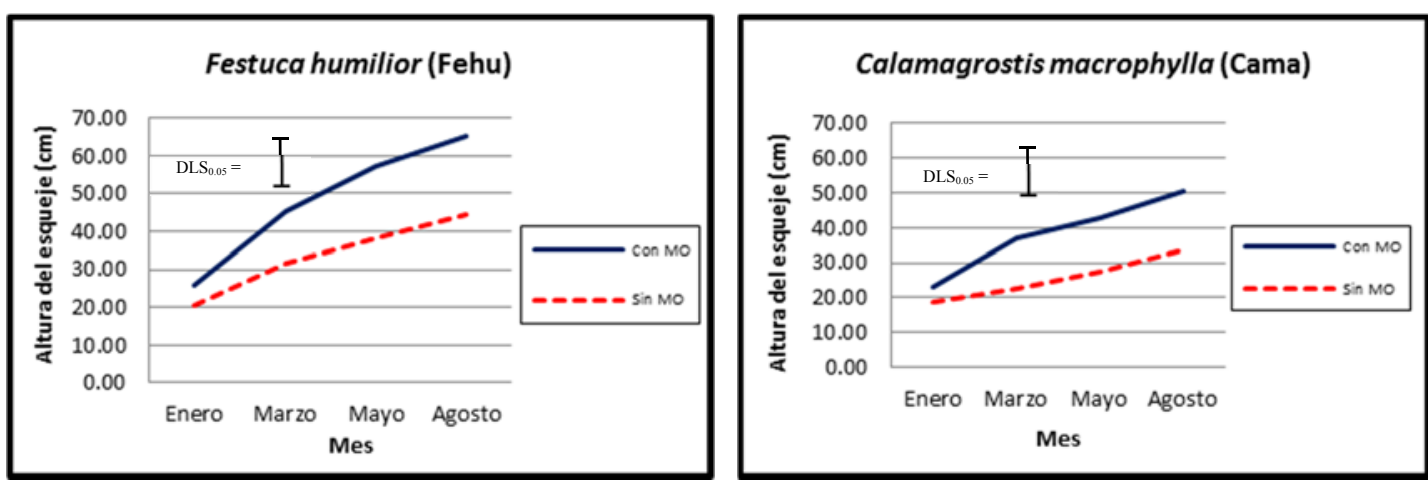

Figura 2. Altura de esquejes con y sin materia orgánica. 

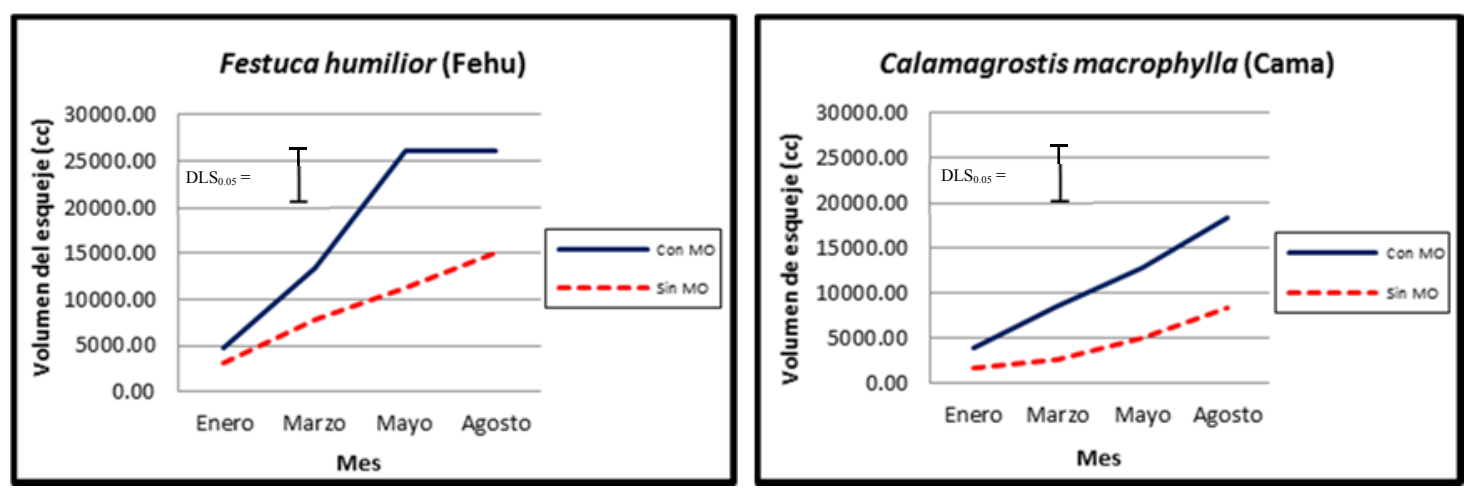

Figura 3. Volumen de esquejes con y sin materia orgánica.

orgánica respectivamente.

Una mejor respuesta en altura y volumen son rasgos ventajosos para los esquejes trasplantados, debido a que pueden aumentar su capacidad competitiva frente a otras especies nativas que coexisten en simultáneo (Fehr, 1987). Estos rasgos incluyen un crecimiento rápido durante la época lluviosa (Schröder \& Prasse, 2013), una mayor área basal (Wilsey, 2010) y mayor biomasa aérea (Lambert et al., 2011). El vigor de ambas especies propone que los rasgos por los que fueron seleccionados permiten una mayor captación de recursos y tener una mayor ventaja competitiva frente a otras especies con crecimiento tardío (Gustafson et al., 2004).

Nuestros resultados son acordes a los trabajos realizados por Mola et al. (2011), Jiménez et al. (2013) y Abella \& Smith (2013) quienes encontraron una respuesta positiva a la revegetación con plantas nativas, expresado con un mejor vigor, situación que fue favorecida por la disponibilidad de micrositios fértiles para el desarrollo de los esquejes, los cuales estaban enriquecidos con nutrientes que provee la adición de la materia orgánica. Este microclima mejoró la actividad biológica del suelo y la aparición posterior de plantas anuales, lo cual fue un indicativo de la mejora del estatus ecológico del suelo, considerado como primer paso para la restauración del ecosistema degradado (Padilla \& Pugnaire, 2006).
Respuesta Hidrológica del Pastizal

Los resultados demostraron que la tasa de infiltración terminal mejoró notablemente con la adición de materia orgánica de 0.1 a $0.2 \mathrm{~cm} / \mathrm{min}$. $(\mathrm{P}=0.04)$ (Figura 4). Diferenciando esta respuesta entre ambas especies, se encontraron mayores tasas de infiltración terminal con la revegetación con Festuca humilior $(0.15 \mathrm{~cm} / \mathrm{min}$.), mostrando una ligera tendencia de mejora en comparación con la revegetación con Calamagrostis macrophylla (0.12 $\mathrm{cm} / \mathrm{min})(\mathrm{P}=0.04)$, siendo ambas superiores a lo encontrado en las tierras de usufructo comunal control $(0.10 \mathrm{~cm} / \mathrm{min})(\mathrm{P}<0.05)$.

Asociado con una mayor infiltración, el contenido de humedad del suelo (Figura 5) mostró una tendencia favorable cuando se incorporó la materia orgánica en los campos revegetados $(\mathrm{P}=0.04)$ (Mwendera \& Saleem, 1997). En el caso de la Festuca humilior se encontraron porcentajes finales de humedad del suelo de $27.8 \%$ y $15.7 \%$ para los tratamientos con y sin materia orgánica respectivamente. La misma tendencia se encontró en el caso de la Calamagrostis macrophylla con porcentajes finales de humedad del suelo de $24.2 \%$ y $13.8 \%$ para los tratamientos con y $\sin$ materia orgánica respectivamente. Todos los tratamientos, superaron los porcentajes finales de humedad del suelo de las tierras de usufructo comunal (Usufructo: 11.5\%).
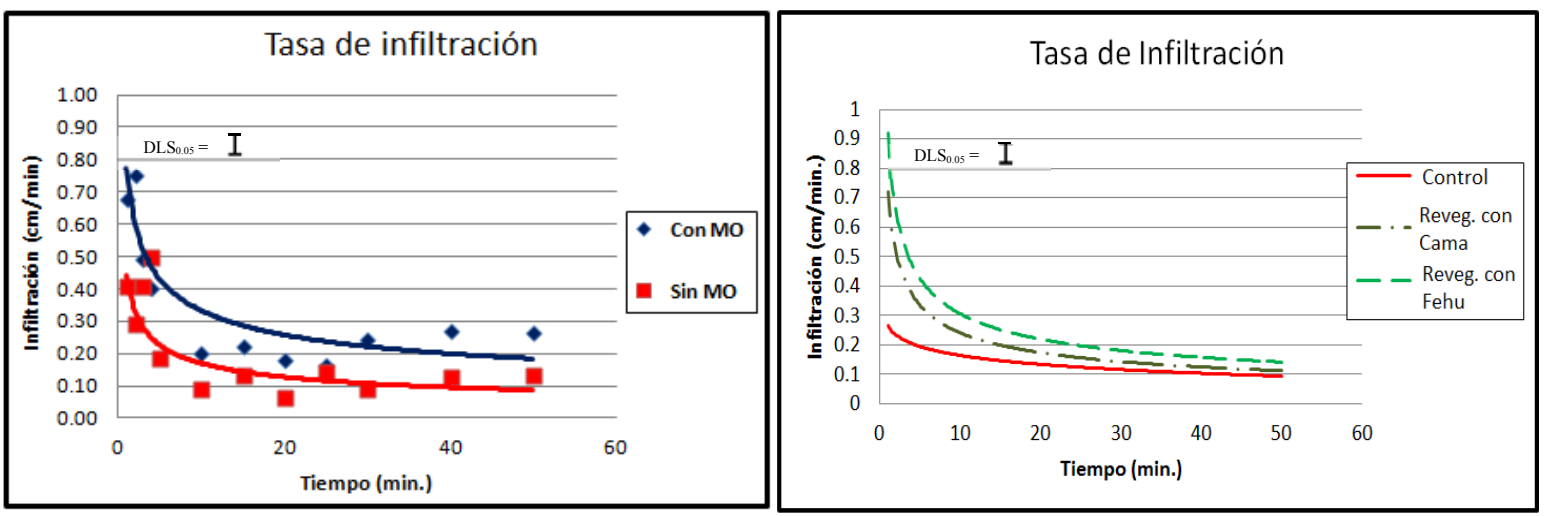

Figura 4. Tasa de infiltración terminal por tratamiento $(\mathrm{cm} / \mathrm{min}$.). 


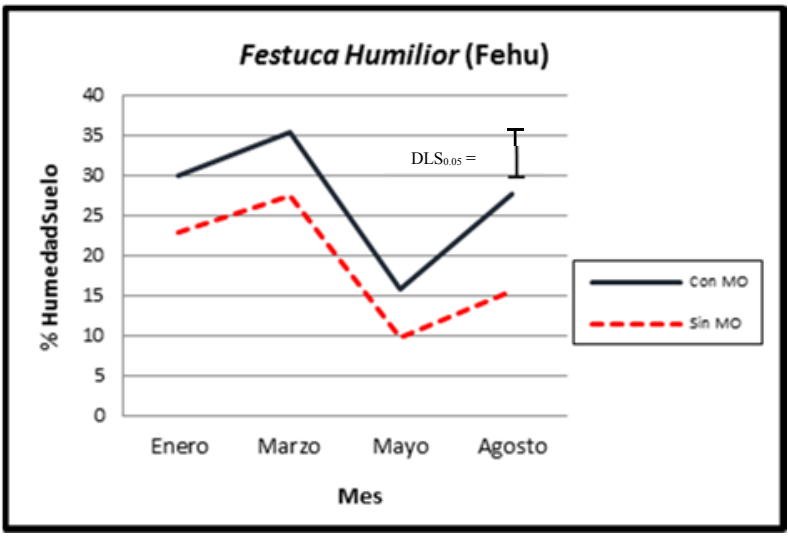

Figura 5. Humedad del suelo por tratamiento (\%).

Este porcentaje de humedad obtenido en los tratamientos con materia orgánica (T1 y T3) podrían haberse favorecido por la generación de microrelieves a nivel superficial que contribuyan a retener humedad $\mathrm{y}$ favorecer la infiltración, tal como lo reportado por Hoogendoorn et al. (2015), quienes encontraron que la aparición de microrelieves a diferentes intensidades de pastoreo, tiene una influencia dominante sobre la acumulación neta anual de forraje, contenido de humedad, composición química del suelo y niveles de lixiviación de Nitrógeno y Carbono del suelo.

La respuesta encontrada para la infiltración y humedad del suelo demostró el rol de la vegetación y la materia orgánica sobre la protección del suelo, lo cual mejoró sus propiedades físicas, reduciendo la escorrentía superficial e incrementando la infiltración y la retención de humedad en el suelo (Wild, 1992; Pierson et al., 2002). Asimismo, la materia orgánica protege la superficie del suelo ante perturbaciones ambientales, favorece el desarrollo y actividad de los microorganismos del suelo incrementando la aireación y la penetrabilidad de la raíz, situaciones que puede mejorar aún más el estado hídrico de la comunidad (Gusquiani et al., 1995; Ghose, 2001).

Los resultados sugieren que la respuesta hidrológica a la revegetación fue favorecida por la incorporación de materia orgánica, lo cual puede deberse a la influencia sobre la estabilidad de los agregados del suelo (Sun et al., 1999 y Zhang \& Horn, 2001). La materia orgánica se compone de un pool de iones tanto activos como pasivos que favorecen la dinámica del carbono dentro del suelo y los nutrientes, condición que favorece la formación $y$ estabilización de los macroagregados y microagregados. Al respecto, Attou et al. (1998) reportan que la adición de materia orgánica afecta físicamente la agregación a través de la apertura de

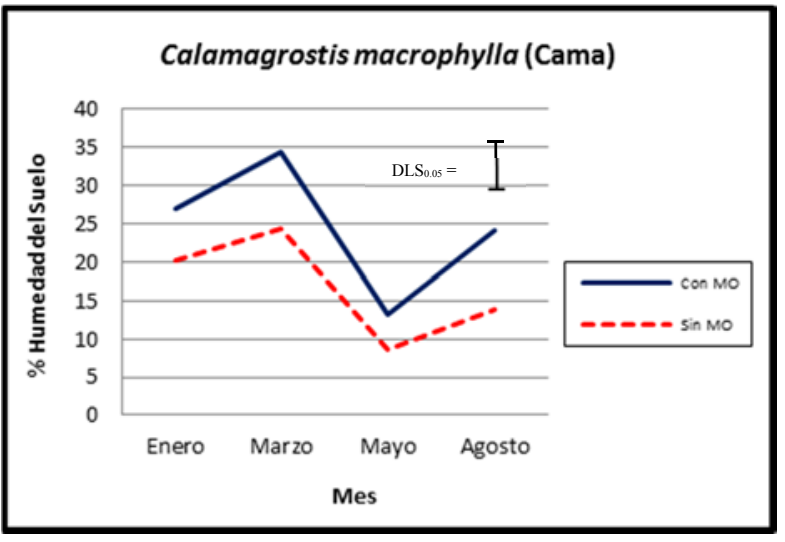

macroporos y la dispersión, lo cual influye en varios aspectos de la conducta física del suelo, tales como la infiltración del agua y la erosión del suelo. Asimismo, se debe considerar que el establecimiento exitoso de los esquejes resulta en un incremento de la cubierta vegetal que reduce la evaporación del suelo y en consecuencia el aumento de la humedad del suelo (Suding et al., 2004; Bezemer et al., 2006; Kardol \& Wardle, 2010).

Condición Ecológica del Pastizal

La condición ecológica del pastizal mejoró significativamente de pobre a regular con la aplicación de los tratamientos (Usufructo: 31.6\%, T1: 52.8\%, T2: 51.7\%, T3: $51.9 \%$ y T4: $51.1 \%$, respectivamente) en comparación con las áreas de usufructo comunal como resultado del efecto que tiene la revegetación en la reducción de suelo desnudo, (Usufructo: 37.0\%, T1: $9.0 \%$, T2: $9.0 \%$, T3: $10.0 \%$ y $\quad$ T4: $11.0 \%$, respectivamente). Respuesta similar se observó en la proporción de especies deseables para ovinos (Usufructo: $13.0 \%, \mathrm{~T} 1: 31.0 \%$, T2: 30.0\%, T3: 32.0\% y T4: $32.0 \%$, respectivamente), el incremento en especies deseables favoreció el incremento del índice forrajero (Usufructo: $49.0 \%$, T1: $78.0 \%$, T2: 76.0\%, T3: $75.0 \%$ y T4: $73.0 \%$, respectivamente) (Tabla 1). $\mathrm{Si}$ bien los porcentajes en condición fueron similares entre ambas especies, en el caso de las parcelas revegetadas con Festuca humilior se observó un mejor índice de vigor que las revegetadas con Calamagrostis macrophylla (T1: $34.7 \%$, T2: $32.5 \%$ frente a T3:

Tabla 1. Condición ecológica por tratamiento.

\begin{tabular}{lccccc}
\hline Condición Ecológica & $\begin{array}{c}\text { Usufructo } \\
\text { (Control) }\end{array}$ & $\begin{array}{c}\text { T1: } \\
\text { Fehu } \\
\text { MO }\end{array}$ & $\begin{array}{c}\text { T2: } \\
\text { Fehu }\end{array}$ & $\begin{array}{c}\text { T3: } \\
\text { Cama } \\
\text { MO }\end{array}$ & $\begin{array}{c}\text { T4: } \\
\text { Cama }\end{array}$ \\
\hline Deseables (\%) & 13.0 & 31.0 & 30.0 & 32.0 & 32.0 \\
Índice forrajero (\%) & 49.0 & 78.0 & 76.0 & 75.0 & 73.0 \\
Indeseables (\%) & 1.0 & 0.0 & 1.0 & 0.0 & 4.0 \\
Suelo Desnudo (\%) & 37.0 & 9.0 & 9.0 & 10.0 & 11.0 \\
Índice de Vigor (\%) & 27.3 & 34.7 & 32.5 & 28.5 & 27.7 \\
Mantillo (\%) & 9.0 & 14.0 & 12.0 & 11.0 & 12.0 \\
Puntaje (\%) & 31.6 & 52.8 & 51.7 & 51.9 & 51.1 \\
Condición (Ovinos) & Pobre & Regular & Regular & Regular & Regular \\
\hline
\end{tabular}


$28.5 \%$ y T4: $27.7 \%$ ), lo cual podría deberse a las diferencias inherentes a la morfología y fisiología de estas especies así como el grado de respuesta a la revegetación (Willems et al., 1993).

Al finalizar el estudio, se encontró una mayor cobertura vegetal en los campos revegetados (Usufructo: 63.0\%, T1: 91.0\%, T2: 91.0\%, T3: 90.0\% y T4: $89.0 \%$ ), lo que muestra hasta un $28 \%$ más de cobertura vegetal que los campos de usufructo comunal. Los resultados muestran claramente que con la introducción de gramíneas nativas claves vía revegetación por esqueje y la adición de materia orgánica, mejora el valor de los componentes que determinan la condición ecológica del pastizal (Call \& Roundy, 1991; Ratliff, 1993). Estos resultados son consistentes con lo reportado por Jing et al., (2014), quienes observaron que la incorporación de orina y estiércol, mejora el microclima del suelo y por ende la riqueza y diversidad de las plantas. Asimismo, Gao et al. (2002) encontraron que la revegetación mejoró la cobertura vegetal en niveles de hasta un $85.0 \%$ en suelos degradados de Jilantai Salt Lake, China y en el presente estudio, el incremento en cobertura fue de 26 a $28 \%$ con lo que se alcanzó valores que llegaron al $91 \%$ de cobertura.

\section{Conclusiones.}

La revegetación con gramíneas nativas mejoró la cobertura vegetal, sobrevivencia, densidad de plantas, infiltración y contenido de humedad del suelo de pastizales degradados y la respuesta fue mejorada con la adición de estiércol y orina de ganado ovino sugiriendo que es posible manejar el componente animal al pastoreo para maximizar el efecto que este tiene en el ciclo y disponibilidad de nutrientes para un adecuado crecimiento y establecimiento de los esquejes. Estos cambios en los atributos de la vegetación variaron dependiendo de la especie vegetal, se observaron mejores resultados con Festuca humilior que con Calamagrostis macropylla lo cual sugiere la necesidad de diseñar estudios específicos para evaluar el potencial de otras especies en programas de rehabilitación de tierras empobrecidas por el mal manejo y sobrepastoreo. El estudio demostró también que es posible mejorar el estatus ecológico de pastizales pobres en el corto plazo utilizando material vegetativo y contemplando la adición de materia orgánica, así como la participación activa de los comuneros en todas las fases de una investigación que es crucial para lograr el compromiso de ellos en programas futuros de rehabilitación de tierras.

\section{Agradecimientos.}

El presente artículo fue financiado con aporte de la Fundación Mc Knight en colaboración con el Instituto de Montaña y la Comunidad Campesina Cordillera Blanca, Ancash. Los autores agradecen los valiosos comentarios y sugerencias efectuadas al manuscrito por Steven Vanek, University of Cornell y Steven Fonte del International Center for Tropical Agricultura (CIAT).

\section{Literatura citada.}

Abella S.R. \& Smith S.D. 2013. Annual-perennial plant relationships and species selection for desert restoration. Journal of Arid Land. 5, 298-309.

Abella S.R., Chiquoine L.P., Newton A.C. \& Vanier C.H. 2015. Restoring a desert ecosystem using soil salvage, revegetation, and Irrigation. Journal of Arid Environments 115, 44-52.

Allen E.B. 1995. Restoration ecology: limits and possibilities in arid and semiarid lands. In: Roundy B.A., McArthur E.D., Haley J.S. \& Mann D.K. (Eds.), Proceedings: Wildland Shrub and Arid Land Restoration Symposium. General Technical Report INT-GTR-315. U.S. Forest Service, Intermountain Research Station, Ogden, Utah, pp. 7-15.

Amezketa E. 1999. Soil aggregate stability: a review. Journal of Sustainable Agriculture. 14, 83-151.

Attou F., Bruand A. \& Le Bissonnais Y. 1998. Effect of clay content and silt-clay fabric on stability of artificial aggregates. European Journal of Soil Science 49, 569577.

Bezemer T., Lawson C.S., Hedlund K., Edwards A.R., Brook A.J., Igual J.M., Mortimer S.R. \& Van Der Putten W.H. 2006. Plant species and functional group effects on abiotic and microbial soil properties and plant-soil feedback responses in two grasslands. Journal Ecology. 94, 893-904.

Blake G.R. 1965. Bulk Density. In: C. A. Black (ed.). Methods of soil analysis. Part Physical and mineralogical properties, including statistics of measurements and sampling. pp. 374-390.

Bouwer H. 1986. Intake Rate: Cylinder Infiltrometer. En: Page, A.L. R.H. Miller, y D.R. Keeney. Methods of Soil Analysis. Part I. Physical and Mineralogical Methods. Second Edition. Agronomy 9. Madison. Wisconsin, USA, 825-844.

Burke A. 2008. The effect of topsoil treatment on the recovery of rocky plain and outcrop plant communities in Namibia. Journal of Arid Environments. 72, 15311536.

Call C.A. \& Roundy B.A. 1991. Perspectives and processes in revegetation of arid and semiarid rangelands. Journal of Range Management. 44(6), 543-549.

Commander L.E., Rokich D.P., Renton M., Dixon K.W. \& Merritt D.J. 2013. Optimising seed broadcasting and greenstock planting for restoration in the Australian arid zone. Journal of Arid Environments. 88, 226-235.

de la Riva E.G., Casado M.A., Jiménez M.D., Mola I., Costa-Tenorio M. \& Balaguer, L., 2011. Rates of local colonization and extinction reveal different plant community assembly mechanisms on road verges in central Spain. Journal of Vegetation Science. 22, 292302.

Dregne H.E. \& Chou N.T. 1992. Global desertification dimensions and costs. In: Dregne, H.E. (Ed.), Degradation and Restoration of Arid Lands. Lubbock: Texas Technical University.

Flores E.R. 1996. Realidad, limitaciones y necesidades de investigación del sector pecuario peruano. Latin 
America livestock regional assessment workshop. San Jose de Costa Rica Published by the Management Entity, Small Ruminant CRP, University of California, Davis, California 83-96.

Flores E.R. 1999. Tambos alpaqueros y pastizales II: Mejoramiento de praderas naturales. Proyecto especial tambos alpaqueros. Boletín técnico LUP $\mathrm{N}^{\circ}$ 12. Lima, Perú.

Gao Y., Yu Qiu G., Shimizu H., Tobe K., Sun B. \& Wang, J. 2002. A 10-year study on techniques for vegetation restoration in a desertified salt lake area. Journal of Arid Environments. 52, 483-497.

Ghose M.K. 2001. Management of topsoil for geoenvironmental reclamation of coal mining areas. Environmental Geology. 40, 1405-1410.

Gillen R.L. \& Tate K.W. 1993. The constituent differential method for determining live and dead herbage. Journal of Range Management. 46(2), 142-147.

Fehr W.R. 1987. Principles of Cultivar Development. Macmillan Publishing Company, New York, NY.

Flores E.R. \& Ñaupari J.A. 2011. Identificación y evaluación de ecosistemas de la cuenca del Río Santa con el uso de Sistemas de Información Espacial. Convenio UICN-UNALM. Informe publicado por el Laboratorio de Ecología y Utilización de Pastizales UNALM.

Gusquiani P.L., Pagliai M., Gigliotti G., Businelli D. \& Benetti A. 1995. Urban waste compost: effects on physical, chemical and biochemical soil properties. Journal of Environmental Quality. 24,175-82.

Gustafson D.J., Gibson D.J. \& Nickrent D.L. 2004. Competitive relationships of Andropogon gerardii (Big Bluestem) from remnant and restored native populations and select cultivated varieties. Functional Ecology. 18, $451-457$.

Herbel C.H. 1983. Principles of intensive range improvements. Journal of range management. 36(2):140-144.

Hoag J.C. 2003. Harvesting, Propagating, and Planting Wetland Plants. USDA Natural Resources Conservation Service, Boise, Idaho. TN Plant Materials No. 13.

Hoogendoorn C.J., Newton P.C.D., Devantier B.P., Rolle B.A., Theobald P.W. \& Lloyd-West C.M. 2015. Grazing intensity and micro-topographical effects on some nitrogen and carbon pools and fluxes in sheep-grazed hill country in New Zealand. Agriculture, Ecosystems and Environment. 217, 22-32.

Hulbert S.H. 1984. Pseudoreplication and the design of ecological field experiments. Ecological Monographs. $54,187-211$.

Hurtado L. 2007. Fundamentos de riego. Publicación UNALM. Facultad de Agronomía. Artículo en línea disponible en www.pdffactory.com.

Ilunga wa Ilunga E., Mahy G., Piqueray J., Séleck M., Shutcha M. N., Meerts P. \& Faucon M. 2015. Plant functional traits as a promising tool for the ecological restoration of degraded tropical metal-rich habitats and revegetation of metal-rich bare soils: A case study in copper vegetation of Katanga, DRC. Ecological Engineering. 82, 214-221.

Jiménez M.D., Ruiz-Capilla P., Mola I., Pérez-Corona E., Casado, M.A. \& Balaguer, L. 2013. Soil development at the roadside: a case study of a novel ecosystem. Land Degradation \& Development. 24, 564-574.
Jing Z.B., Cheng J.M., Su J.S., Bai Y. \& Jin J.W. 2014. Changes in plant community compostion and soil properties under 3-decade grazing exclusion in semiarid grassland. Ecological Engineering. 64, 171-178.

Kardol P.\& Wardle D.A. 2010. How understanding aboveground-belowground linkages can assist restoration ecology. Trends in Ecology and Evolution. 25, 670-679.

Krogh S.N., Zeisset M.S., Jackson E. \& Whitford W.G. 2002. Presence/absence of a keystone species as an indicator of rangeland health. Journal of Arid Environments. 50, 513-519.

Laboratorio de Análisis de Suelos y Aguas UNALM. 2011. Resultados de caracterización físico-química de suelos.

Lambert A.M., Baer S.G. \& Gibson D.J. 2011. Intraspecific variation in ecophysiology of three dominant prairie grasses used in restoration: cultivar versus non-cultivar population sources. Restoration Ecology. 19, 43-52.

Lawesson J.E. \& Oksanen J. 2002. Niche characteristics of Danish woody species as derived from coenoclines. Journal of Vegetation Science. 13, 279-290.

Mamani G. 2002. Zonificación ecológica para la aplicación de estrategias de mejoramientos de praderas naturales de la microcuenca del Río Negro, Ancash. Tesis de Maestría en Producción Animal, UNALM. Lima, Perú.

Méndez M., García D., Maestre F.T. \& Escudero A. 2008. More ecology is needed to restore mediterranean ecosystems: a reply to Valladares and Gianoli. Restoration Ecology. 16 (2), 210-216.

Moreno, A. 2015. Actividades de riesgo, abonado y tratamiento en cultivos. FPB Agraria. España.

Mola I., Jiménez M.D., López-Jiménez N., Casado M.A. \& Balaguer L., 2011. Roadside reclamation outside the revegetation season: management options under schedule pressure. Restoration Ecology. 19, 83-92.

Montemayor M., Price J., Rocherfort L. \& Boudreau S. 2008. Temporal variations and spatial patterns in saline and waterlogged peat fields: 1 . Survival and growth of salt marsh graminoids. Environmental and Experimental Botany. 62, 333-342.

Mwendera E.J. \& Saleem M.A. 1997. Hydrologic response to cattle grazing in the Ethiopian highlands. Agriculture, Ecosystems \& Environment. 64(1), 33-41.

Padilla F.M. \& Pugnaire F.I. 2006. The role of nurse plants in the restoration of degraded environments. Frontiers in Ecology and the Environment. 4, 196-202.

Parker K.W. 1951. Method of measuring trend in range conditions on national forest ranges. USDA Forest Service, $26 \mathrm{pp}$, mixeo.

Petersen S. \& Stringham T. 2008. Infiltration, runoff, and sediment yield in response to western juniper encroachment in southeast Oregon. Journal of Rangeland Ecology and Management. 61, 74-81.

Pierson F.B., Spaeth K.E., Weltz M.A. \& Carlson D.H. 2002. Hydrologic response of diverse western rangelands. Journal of Range Management. 55, 558570.

Pyke D.A., Herrick J.E., Shaver P. \& Pellant M. 2002. Rangeland Health Attributes and Indicators for Qualitative Assessment. Journal of Range Management. 55, 584-297.

Ratliff R.D. 1993. Viewpoint: Trend assessment by similarity - a demonstration. Journal of Range Management. 46, 139-141. 
Rodríguez V. 2006. El majadeo o redileo. Revista Fertilidad de la Tierra $\mathrm{N}^{\circ}$ 23. Pág. 63-66.

Rondán V. \& Chávez D.E. 2014. Estudio de manadas de la comunidad campesina Cordillera Blanca. Publicación del Instituto de Montaña, Huaraz- Perú.

Schwinning S. \& Hooten M.M. 2009. Mojave Desert root systems. In: Webb R.H., Fenstermaker L.F., Heaton J.S., Hughson D.L., McDonald E.V. \& Miller D.M. (Eds.). The Mojave Desert: Ecosystem Processes and Sustainability. University of Nevada Press, Reno, USA, pp. 278-311.

Schröder R. \& Prasse R. 2013. Cultivation and hybridization alter the germination behavior of native plants used in revegetation and restoration. Restoration Ecology. 21,793-800.

Smith S.D., Monson R.K. \& Anderson J.E. 1997. Physiological Ecology of North American Desert Plants. Springer, New York, USA.

Snyman H.A. \& Du Preez C.C. 2005. Rangeland degradation in a semi-arid South Africa, II: influence on soil quality. Journal of Arid Environment. 60, 483-507.

Steed, J.E. \& DeWald L.E. 2003. Transplanting sedges (Carex spp.) in southwestern riparian meadows. Restoration Ecology. 11, 247-256.

Suding K. N., Gross K. L. \& Houseman G. R. 2004. Alternative states and positive feedbacks in restoration ecology. Trends in Ecology and Evolution. Vol.19 No.1.

Sun B., Zhang T.L. \& Zhao Q.G. 1999. Fertility evolution of red soil derived from quaternary red clay in low-hilly region in middle subtropics: I. Evolution of soil physical fertility. Acta Pedol. Sin. (in Chinese). 36(1), 35-47.
Thomsen D., Marsden I.D. \& Sparrow A.D. 2005. A field experiment to assess transplant success of salt marsh plants into tidal wetlands. Wetlands Ecology and Management. 13, 489-497.

Weaver J.E. \& Darlan R.W. 1947. A method of measuring vigor of range grasses. University of Nebraska Lincoln. Agronomy and Horticulture Department Ecology. 28(2), 146-162.

Whitford W.G. 1995. Desertification: implications and limitations of the ecosystem health metaphor. In: Rapport, D.J., Gaudet, C. L. \& Calow, P. (Eds), Evaluating and Monitoring the Health of Large-Scale Ecosystems, pp. 257-166. NATO ASI Series. Berlin: Springer-Verlag.

Wild A. 1992. Condiciones del suelo y desarrollo de las plantas según Russell. Versión Española de P. Urbano Terrón y C. Rojo Fernández. Mundi-Prensa. Madrid, España. 1045 pp.

Willems J.H., Peet R.K. \& Bik L. 1993. Changes in chalkgrassland structure and species richness resulting from selective nutrient additions. Journal of Vegetation Science. 4, 203-212.

Willms W., McGinn S. \& Dormaar J. 1993. Influence of litter on herbage production in the mixed prairie. Journal of Range Management. 46, 320-324.

Wilsey B.J. 2010. Productivity and subordinate species response to dominant grass species and seed source during restoration. Restoration Ecology. 18, 628-637.

Zhang B. \& Horn R. 2001. Mechanisms of aggregate stabilization in Ultisols from subtropical China. Geoderma. $\quad 99,123-145$.

\footnotetext{
${ }^{1}$ Investigador Asociado. Laboratorio de Ecología y Utilización de Pastizales, Departamento de Producción Animal de la Facultad de Zootecnia, Universidad Nacional Agraria La Molina, Av. La Molina s/n La Molina, Lima, Casilla Lima 12, Perú, lab pastizales@lamolina.edu.pe.

2 Profesor Principal. Laboratorio de Ecología y Utilización de Pastizales, Departamento de Producción Animal de la Facultad de Zootecnia, Universidad Nacional Agraria La Molina, Av. La Molina s/n La Molina, Lima, Casilla Lima 12, Perú, lab_pastizales@lamolina.edu.pe.
} 